\title{
The Research on the Pre Blocking Scheduling Method for Elevated Road Based on Video
}

\author{
Rongbao Chen ${ }^{1}$, Yang Liu ${ }^{1}$, Qianlong Wang, Honghui Jiang and Dawei Tang \\ Academy of electrical and automation engineering, Hefei University of Technology, Hefei 230009, Anhui Province China \\ ${ }^{*}$ Corresponding author
}

\begin{abstract}
This paper has obtained the taxi speed through the high road video monitoring system to determine whether the elevated road is congested. A scheduling method is proposed to preclude overhead road congestion in advance. The existing video monitoring system is used as base. The vehicle is extracted by means of motion target detection. The type of vehicle is distinguished by the $\mathrm{Hu}$ invariant moment. The vehicle is divided into color and non-color through HSL (Hue Saturation and Lightness) space. The centroid location of the color vehicle is carried out and the color recognition of the matrix in the centroid range is used to judge whether the vehicle has a dome light. If the vehicle is a taxi, measure the speed of the taxi. Traffic congestions in the viaduct is deemed to occur soon if the taxi speed is obviously lower. Relevant measures shall be taken immediately to eliminate the possibility of congestion and ensure the normal use of viaduct. Because of the complexity of traffic congestion, the process of removing congestion is very difficult. Predicting the occurrence of traffic congestion and scheduling can alleviate this contradiction. Meanwhile it improves the intelligent property of video surveillance system and saves social resources.
\end{abstract}

Keywords-visual detection; viaduct; taxi speed; pre exclude congestion

\section{INTRODUCTION}

The development of urban traffic changes with each passing day with the progress of science and technology. The urban traffic jams become more and more serious because of the limitations of urban planning and the increase of the number of motor vehicles [1]. The viaduct system is born in order to alleviate traffic congestion and that plays an important role in the existing urban road transportation system. The traffic condition of the main line of viaduct is becoming worse with the rapid increase of traffic flow and the deterioration of traffic congestion [2]. The situation is even more serious when congestion occurs because the viaduct has a certain closeness. It is usually through artificial observation to complete the monitoring or control tasks for the viaduct road congestion at present. In case of abnormal situation, alarm and road information system will be prompted. This will consume a lot of manpower and material resources and lack of intelligence [3]. Therefore, it is urgent to develop a real-time video surveillance, feedback and disposal system [4].

It is first of all to accurately and timely judge and handle the traffic events on the viaduct to ensure the normal use of viaduct. Many video surveillance cameras are installed on elevated roads including the entrance of ramp for the existing road video surveillance system. Judge the congestion of the elevated road quickly and accurately by collecting and analyzing video information of each camera in real time. This method has advantages compared with other highway congestion monitoring methods [5]. The video surveillance system is perfect and the system design cost is low. Judge the situation of elevated road quickly and accurately with collecting road traffic flow in real time. Installation and maintenance is simple will not destroy the road surface. It is suitable for all-weather monitoring and disposal. Early warning mechanism can avoid the delay of congestion monitoring system [6]. It is important for the vehicle trip guidance that the timely feedback and intelligent disposal of the system.

\section{FEATURE SELECTION IN VideO Detection OF ELEVATED ROAD}

The existing technologies for analyzing urban traffic congestion include the loop coil method, the radar (microwave) method, the ultrasonic method and the method based on GPS data and so on. The loop coil method is inconvenient to install and affects the life of road surface, so it has a large identification error. The radar (microwave) method and the ultrasonic method cannot detect stationary or low-speed vehicles, and the detection performance decreases with the influence of temperature and airflow [7]. The method based on the GPS data may lead to signal detection blind areas, and to obtain data large-scale installation of GPS device vehicles is needed. The video processing technology has many advantages, such as large amount of information processing, no need to destroy the ground, convenient installation and maintenance. It has a good application prospect in road traffic congestion analysis and discrimination [8].

As a kind of urban public transportation, taxi has the characteristics of convenience and rapidness, and it provides convenience for people to travel. Thus taxis may randomly appear anywhere in the city. Qualification regulations of the taxi ensure the violation records and minimum taxi accident record. The data show that the taxi drivers drive 10-12 million kilometers average annual [9]. In 2015 the national death rate of taxi traffic accident is 0.47 per hundred million kilometers, while more than 11000 taxis in Hefei, the number of deaths related to taxi accidents is no more than $1 \%$ of the total number of deaths from traffic accidents in 2015 [10].

The number of vehicles on the elevated road is very high and the speed is fast. At present, the detection and research of 
traffic congestion at home and abroad is not mature enough, the algorithm complexity is high, and the practical application is difficult. As shown in Figure 1, the current highway video surveillance system has been quite perfect. And the video surveillance cameras are installed on the elevated road ramp including corners and other locations. Taking Hefei city as an example, the distance between the cameras is about 500-700 meters, to monitor traffic conditions of the entire viaduct. Because of the high proficiency of taxi drivers, the urban expressway can be maintained at a constant speed, and the speed of the upper and lower viaducts is not more than $40 \mathrm{~km} / \mathrm{h}$. The congestion in elevated road is usually caused by occasional accidents or vehicle surges, and these two causes have significant impact on speed of taxi. If an accident occurs, the speed of the taxi in front of the viaduct is not affected, and the speed of the taxi will fall behind the accident site. If the number of vehicles on the viaduct increases sharply, the taxi speed will slow down at the same time [11]. For the current video surveillance system, the characteristics of the taxi type, vehicle color and position of dome light are obvious and easy to identify, so as to meet the needs of all-weather monitoring. Therefore, the speed of the taxi can be used as a pre-judgment for the congestion of elevated roads.

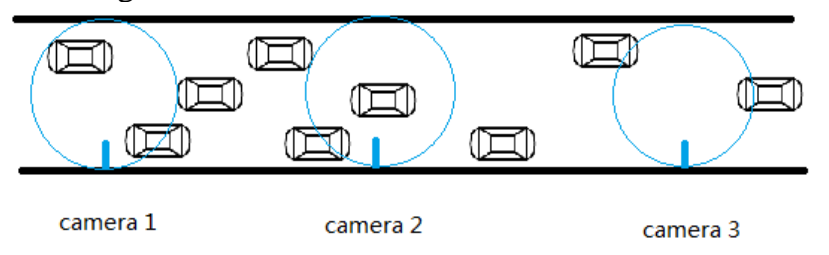
FIGURE I. THREE-DIMENSIONAL COORDINATES AND THE
GROUND COORDINATES OF THE AIRCRAFT

\section{TAXI IDENTIFICATION IN VIDEO SURVEILLANCE}

The taxi has obvious characteristics. Taking Hefei city as an example, taxis are unified into sedan type. Meanwhile the model of taxi dome light is unified and the main body is white, that installation is in the middle of the vehicle roof. In order to distinguish other types of vehicles, the taxi body is not white.

\section{A. Vehicle Target Detection}

\section{(1) Background extraction}

In the real-time traffic image sequence, a group of pictures of the background can be divided into fixed area stationary region and moving region two parts. The system only needs to deal with the road area.

The system uses the method of the average value of the method to extract the background model. The experiment results show that the more images to get the background of the effect is better, as shown in figure 2. Its principle can be expressed by (1):

$$
B I(x, y)=\frac{\sum_{i=1}^{j} P I_{i}(x, y)}{n} .
$$

In the image sequence, $P I_{i}(x, y)$ is the pixel value of the point $(x, y)$ in the image of the I frame in the type (1). $B I(x, y)$ represents the pixel value of the point $(x, y)$ position of the background image [12].

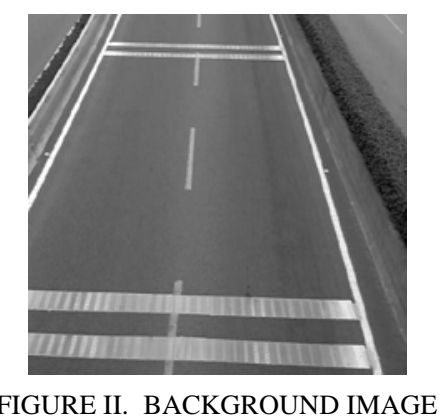

(2) Identification of vehicle targets using an improved hybrid Gauss background modeling algorithm

The mixed Gauss background model uses the statistical information of the pixel samples to represent the background. Assume that the pixel values of R, G, and B three channels are independent and the variance is the same in dealing with RGB color images. The random variable $X$ is used to represent the value of the pixel. Make up a data set as $\left\{X_{1}, X_{2}, \ldots, X_{t}\right\}$ by the random variable $x$ at each moment. Describe by $K$ Gauss distribution model. The pixel sample of the $t$ moment is $X_{t}=\left(r_{t}, g_{t}, b_{t}\right)$.The probability function of the current pixel value is

$$
\left\{\begin{array}{c}
P\left(X_{t}\right)=\sum_{i=1}^{K} \omega_{i, t} * \eta\left(X_{t}, \mu_{i, t}, \sum_{i, t}\right) \\
\eta\left(X_{t}, \mu, \sum\right)=\frac{1}{(2 \pi)^{\frac{n}{2}}\left|\sum\right|^{\frac{1}{2}}} e^{-\frac{1}{2}\left(X_{t}-\mu_{t}\right)^{T} \Sigma^{-1}\left(X_{t}-\mu_{t}\right)} \\
\sum_{i, t} \sigma_{i, t}^{2} I
\end{array} .\right.
$$

In the formula, the $K$ is the quantity of Gauss distribution. The $\eta\left(X_{i}, \mu_{i, t}, \tau_{i, t}\right)$ is the $i$ Gauss distribution of the $t$ moment. $\omega_{i, t}, \mu_{i, t}$, and $\sigma_{i, t}$ are weight coefficients, mean and variance, respectively. The $I$ is the unit matrix.

The foreground is extracted and processed with the background difference method after extracting the foreground. Make it the foreground is strengthened. This makes the foreground stronger. Identify the vehicle through that the gray scale, the prospect of sharpening, filtering and opening operation. Fill all the holes of the vehicle target. The resulting target block is shown in Figure 3. 

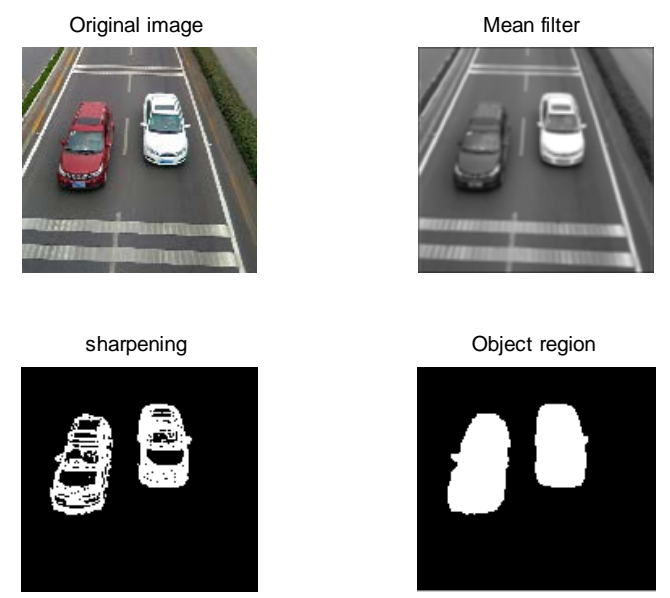

FIGURE III. THE VEHICLE TARGET EXTRACTION

\section{B. The Hu moment algorithm distinguishes vehicle types}

The moment is a rough feature obtained by integrating operations on all points on the contour. This is the simplest way to compare the two contours.

This is the definition of the Hu moment [13]. Set a twodimensional continuous random function. The $m_{p q}$ matrix of the probability density distribution function $f(x, y)$ is defined as $(p+q)$ :

$$
m_{p q}=\int_{-\infty}^{\infty} \int_{-\infty}^{\infty} x^{p} y^{q} f(x, y) d x d y
$$

Define $g(i, j)$ is a digital image. Its two-dimensional moments are

$$
m_{p q}=\sum_{j=0}^{M-1} \sum_{i=0}^{N-1} i^{p} j^{q} g(i, j) .
$$

It is complete set should include all $m_{p q}$ that $p+q \leq n$ for $\mathrm{n}$ order geometric moments. It includes the $\frac{1}{2}(n+1)(n+2)$ elements. The basal function is defined as the product of a single $x^{p} y^{q}$ for this moment. The gray value of the image density distribution function was regarded as a two-dimensional or $\mathrm{H}$ dimension and then the image features can be get by moment method according to the discussion of $\mathrm{Hu}$. The shape feature of the image is represented by an infinite set of transform coefficients $m_{p q}$ after transformation.

The image function is mapped to a two-dimensional polynomial basis function and then the coefficient can be get [14]. The central moment of order $(p+q)$ for the continuous image is considered to be the corresponding image function:

$$
\eta_{p q}=\int_{-\infty}^{\infty} \int_{-\infty}^{\infty}(x-\bar{x})^{p}(y-\bar{y})^{q} f(x, y) d x d y .
$$

In the formula, $\bar{x}=\frac{M_{10}}{M_{00}}, \bar{y}=\frac{M_{01}}{M_{00}}$.

The discrete image that size is $(\mathrm{M} \times \mathrm{N})$ is denoted by $f(x, y)$. The $f(x, y)$ can be written in a moment:

$$
M_{p q}=\sum_{j=1}^{M} \sum_{i=1}^{N} i^{p} j^{q} f(i, j)
$$

In the formula, $i \in M, j \in N, p, q$ is constant.

Make it $\mu_{p q}=\frac{M_{p q}}{M_{00}^{r}}$ and $r=\frac{(p+q+2)}{2}$. Hu got the 7 moment is invariant to rotation, zoom and displacement after transformation:

$$
\begin{aligned}
& M_{1}=\mu_{20}+\mu_{02} \\
& M_{2}=\left(\mu_{20}+\mu_{02}\right)^{2}+4 \mu_{11} \\
& M_{3}=\left(\mu_{30}-3 \mu_{12}\right)^{2}+\left(3 \mu_{21}-\mu_{03}\right)^{2} \\
& M_{4}=\left(\mu_{30}+\mu_{12}\right)^{2}+\left(\mu_{21}+\mu_{03}\right)^{2} \\
& M_{5}=\left(\mu_{30}-3 \mu_{12}\right)\left(\mu_{30}+\mu_{12}\right)\left[\left(\mu_{30}+\mu_{12}\right)^{2}-3\left(\mu_{21}+\mu_{03}\right)^{2}\right]+\left(3 \mu_{21}-\mu_{03}\right) \\
& \left(\mu_{21}+\mu_{03}\right)\left[3\left(\mu_{30}+\mu_{12}\right)^{2}-\left(\mu_{21}+\mu_{03}\right)^{2}\right] \\
& M_{6}=\left(\mu_{20}-\mu_{02}\right)\left[\left(\mu_{30}+\mu_{12}\right)^{2}-\left(\mu_{21}+\mu_{03}\right)^{2}\right]+4 \mu_{11}\left(\mu_{30}+\mu_{12}\right)^{2}\left(\mu_{21}+\mu_{03}\right) \\
& M_{7}=\left(3 \mu_{12}-\mu_{03}\right)\left(\mu_{30}+\mu_{12}\right)\left[\left(\mu_{30}+\mu_{12}\right)^{2}-3\left(\mu_{21}+\mu_{03}\right)^{2}\right]+\left(\mu_{30}-3 \mu_{12}\right) \\
& \left(\mu_{21}+\mu_{03}\right)\left[3\left(\mu_{30}+\mu_{12}\right)^{2}-\left(\mu_{21}+\mu_{03}\right)^{2}\right]
\end{aligned}
$$

The 7 moment is invariant in displacement, rotation and zoom [15]. From $M_{1}$ to $M_{6}$ is invariant when the image mirror conversion occurred meanwhile the last one will have 180 degree change. Hu presents the uniqueness theorem for that. For a piecewise continuous probability density function $f(x, y)$ if only in $f(x, y)$ limited area within the $(x, y)$ plane has a non-zero value. It means that all moments of $f(x, y)$ are present and can uniquely determine the moment set by a $\left\{M_{p q}\right\}$. On the other hand, $f(x, y)$ can be uniquely determined $\left\{M_{p q}\right\}$.

The study found that the main influence factors of the moment is the coverage of the video surveillance camera and the vehicle position. Moving vehicle imaging has a rotation in the video surveillance camera. The range of rotation angle is clockwise 15 degrees to 15 degrees counterclockwise. Get the sedan image and the non-sedan image from the video surveillance camera. Verify the image rotation invariance after grayscale. As shown in Figure 4, 
Original image

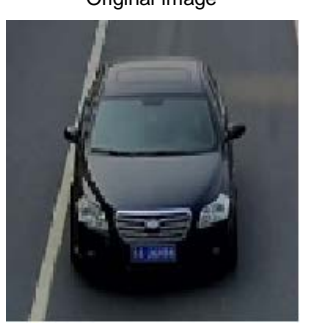

Original image

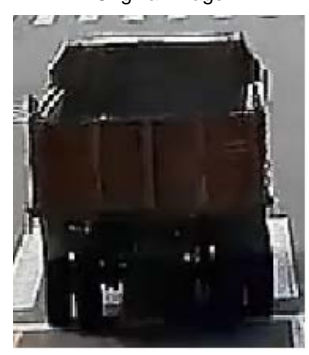

Grayscale image

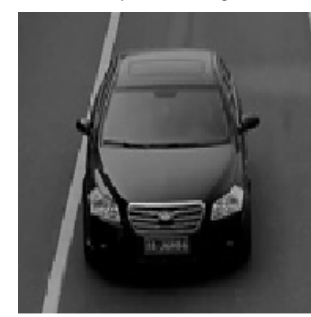

(a)

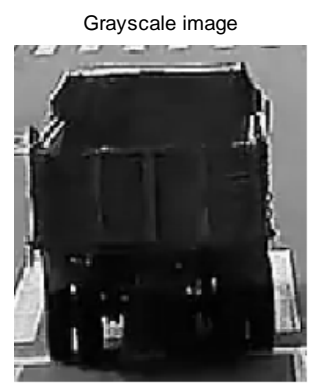

(b)

FIGURE IV. TRUCKS AND CARS IN VIDEO SURVEILLANCE AND THEIR GRAYSCALE PROCESSING

Rotate the grayscale image of figure 4 . The results are shown in table 1 and table 2 . The original image in the table is the grayscale image. The + said clockwise. The - said anticlockwise.

The 7 moment invariants of Hu has rotation invariance by comparison. Therefore, the Hu moment features for vehicle classification is feasible.

Selected sedans and non-sedans models of the 100 samples to determine the feature. The Hu feature vector is the input vector of the support vector machine. The vehicle samples is divided into the sedan and the non-sedans through learning and training. Use the ROC (Receiver Operating Characteristic) curve to evaluate the classification results. It is concluded that the method of the sedan and the non-sedan recognition accuracy rate of up to $99.3 \%$.

TABLE I. SEDAN'S HU MOMENT INVARIANTS

\begin{tabular}{|c|c|c|c|c|c|c|c|}
\hline & $M_{1}$ & $M_{2}$ & $M_{3}$ & $M_{4}$ & $M_{5}$ & $M_{6}$ & $M_{7}$ \\
\hline original image & 6.1000 & 18.3808 & 22.6919 & 21.7315 & 44.1160 & 31.0849 & 49.3141 \\
\hline+5 & 6.1154 & 17.8883 & 22.4228 & 21.7425 & 44.3685 & 30.8489 & 46.6738 \\
\hline+10 & 6.1267 & 17.8057 & 21.8265 & 21.7619 & 44.1831 & 30.8547 & 46.1829 \\
\hline+15 & 6.1384 & 17.7852 & 21.2804 & 21.7951 & 43.8332 & 31.0137 & 44.9626 \\
\hline-5 & 6.1179 & 18.0756 & 22.6578 & 21.6944 & 43.8915 & 30.9140 & 45.5254 \\
\hline-10 & 6.1309 & 17.8569 & 23.4232 & 21.7159 & 44.2867 & 30.8203 & 45.4284 \\
\hline-15 & 6.1421 & $17.62 c 87$ & 24.7269 & 21.7640 & 45.0390 & 30.7449 & 46.1761 \\
\hline
\end{tabular}

TABLE II. NON SEDAN'S HU MOMENT INVARIANTS

\begin{tabular}{|c|c|c|c|c|c|c|c|}
\hline & $M_{1}$ & $M_{2}$ & $M_{3}$ & $M_{4}$ & $M_{5}$ & $M_{6}$ & $M_{7}$ \\
\hline original image & 5.6037 & 13.7359 & 17.5224 & 20.0030 & 38.8937 & 27.0614 & 39.6883 \\
\hline+5 & 5.6451 & 13.7424 & 17.2877 & 19.7417 & 38.4470 & 26.8559 & 40.3465 \\
\hline+10 & 5.6604 & 13.7273 & 17.4076 & 19.8086 & 38.5984 & 26.9722 & 40.5873 \\
\hline+15 & 5.6732 & 13.6821 & 17.4642 & 19.7851 & 38.5822 & 26.9881 & 40.6331 \\
\hline-5 & 5.6278 & 13.5901 & 18.2467 & 20.7017 & 40.3666 & 27.8015 & 40.8419 \\
\hline-10 & 5.6437 & 13.5854 & 18.3227 & 20.8081 & 40.5766 & 27.9890 & 40.9806 \\
\hline-15 & 5.6552 & 13.5565 & 18.3554 & 20.8651 & 40.6930 & 28.1311 & 41.0659 \\
\hline
\end{tabular}

\section{Exterior color}

This paper is based on HSL color space. The color space is a double cone distribution. As shown in Figure 5.

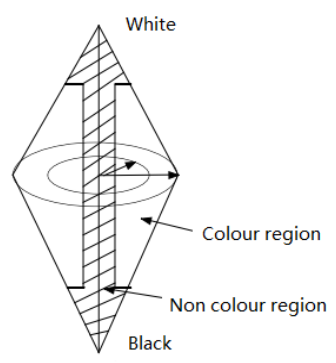

FIGURE V. SKETCH MAP OF HSL COLOR SPACE
The color space is chosen because that can be visually perceived of several common color from the figure. The vertical space coordinates represents lightness. The range is 0 to 1 . That means namely gray from black to white gradient. Angle represents hue. Start from 0 (red) to 120 degrees (green), and then to 240 degrees (blue), and finally back to 360 degrees (red). The radial coordinates is the concentration of color. The distance axis closer, the color is more lighter. The distance axis farther, the color is more concentrated [16].

The actual environment is collected by the image sequence RGB (Red Green Blue) color format. The color image is converted from RGB color space to HSL color space before the vehicle color recognition. Specific methods are as follows. Firstly, R, G, B three components are normalized to the range 
of $[0,1]$. The $\mathrm{H}$ component available type of each pixel is calculated:

$$
H=\left\{\begin{array}{c}
\theta, B \leq G \\
360-\theta, B>G
\end{array}\right.
$$

This:

$$
\theta=\arccos \left\{\begin{array}{c}
\frac{1}{2}[(R-G)+(R-B)] \\
{\left[(R-G)^{2}+(R-B)(G-B)\right]^{\frac{1}{2}}}
\end{array} .\right.
$$

Saturation:

$$
S=1-\frac{3}{(R+G+B)}[\min (R, G, B)]
$$

Hue:

$$
L=\frac{1}{3}(R+G+B)
$$

The $\mathrm{H}$ component range is $[0,360]$ and the $\mathrm{S}$ and $\mathrm{L}$ components are distributed in the range of $[0,1]$ if $R, G, B$ three values were normalized to the range of $[0,1]$.

Between the three components of HSL space are independent of each other. Division of the color area should consider the following points according to the characteristics of the color distribution of double cone:

(1) The $H$ is pointless when the $L$ value is very high or very low.

(2) The $\mathrm{H}$ will not be stable when the $\mathrm{S}$ is very low even near 0 .

(3) The $S$ is pointless when the $L$ value is very high or very low.

The distribution of color in HSL color space show a double cone. The whole color space is divided into non color and color of two kinds of regional according to the relationship of $\mathrm{H}, \mathrm{S}$ and $\mathrm{L}$. The judgment formula of non-color regions are as the formula (12),

$$
\begin{gathered}
\text { White }\left\{\begin{array}{c}
(0.95 \leq L) \\
(0.81 \leq L<0.95) \&\left(S<\frac{18}{180}\right) \\
(0.61 \leq L \leq 0.8) \&\left(S<\frac{20}{180}\right)
\end{array}\right. \\
\text { Black }\left\{\begin{array}{c}
(L<0.25) \\
(0.25 \leq L \leq 0.4) \&\left(S<\frac{60}{180}\right) \\
(0.41 \leq L \leq 0.5) \&\left(S<\frac{40}{180}\right)
\end{array}\right.
\end{gathered}
$$

The rest is judged as a color area. The main color of the taxi is chromatic. The taxi is not white and the black taxi is also very rare taking Hefei city as an example. It is feasible to distinguish the color. The simulation results marked color vehicles with the red color box that might be the target vehicle. The simulation results marked non color vehicles with the blue color box that is the need to remove the vehicle.

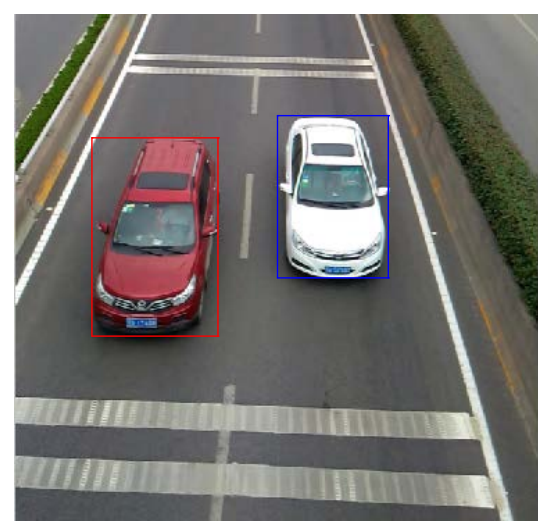

FIGURE VI. VEHICLE COLOR RECOGNITION IN VIDEO
SURVEILLANCE

\section{The Dome Light of Taxi Dome Light Identification}

The dome light of taxi mounted on the middle roof that color is white. There are obvious differences in color and body. The edge detection for color vehicle is got to determine the centroidal coordinates of the vehicle. The centroidal position as the center to set a range of matrix. It means that the vehicle has dome light if the white is detected [17].

The Roberts operator, Laplacian operator, Sobel operator and Canny operator are the commonly used edge detection operator. The Sobel operator is suitable for images with little gray beat and large noise interference. The Roberts operator detection method is applied to images with steep low noise. But the edge positioning accuracy of the Sobel operator and the Roberts operator are not high. The Laplacian operator is very susceptible to noise and therefore does not use much. Canny edge detection is insensitive to noise and has strong anti-interference ability. The effect of the Canny operator is the best for weak edge detection.

The classical Canny edge detection algorithm has three characteristics, such as low false positive rate, high location 
accuracy and false edge suppression. It is very suitable for rough extraction of image edges. The traditional Canny operator uses $2 \times 2$ gradient template to calculate the gradient amplitude. Locate the edge accurately. But it is too sensitive to noise. The $3 \times 3$ template is the template with both accuracy and speed optimum [18]. The edge detection of $\mathrm{X}$ and $\mathrm{Y}$ directions and the gradient calculation of pixels in 45 and 135 degrees directions are as formula (13):

$$
\begin{aligned}
& P_{x}(i, j)=G(i, j+1)-G(i, j-1) \\
& P_{y}(i, j)=G(i+1, j)-G(i-1, j) \\
& P_{45^{\circ}}(i, j)=G(i-1, j+1)-G(i+1, j-1) \\
& P_{135^{\circ}}(i, j)=G(i+1, j+1)-G(i-1, j-1)
\end{aligned}
$$

The gradient amplitude does not remain unchanged if there are 45 and 135 degrees two direction edge in edge detection. So the 8 neighborhood pixels do not have 45 degrees of rotation invariance. This paper uses the formula (14) to calculate the gradient amplitude to solve the problem:

$$
P_{0}^{\circ}(i, j)=\frac{\sqrt{2}-1}{2} P_{x}+\frac{2-\sqrt{2}}{4}\left(P_{45^{\circ}}+P_{135^{\circ}}\right) .
$$

The formula can not only guarantee the edge of the extracted edge, but also can suppress the noise at the same time. The color vehicle is identified with improved Candy edge detection, and the extracted contours are extracted by centroidal coordinate. The simulation results shown in Figure 7.

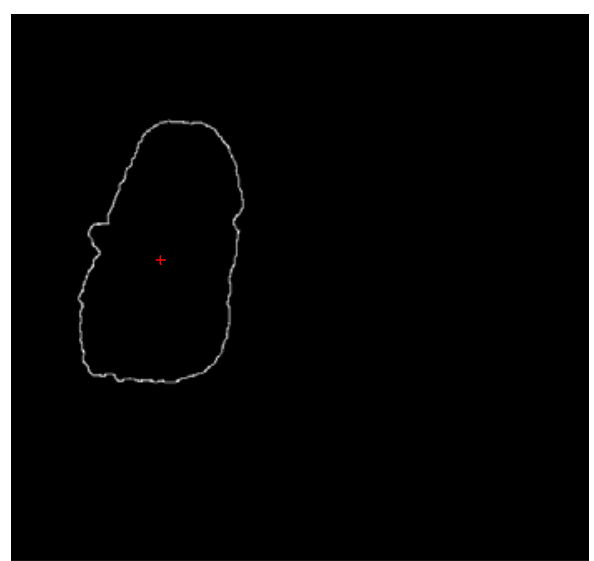

FIGURE VII. THE COLOR OF VEHICLE CENTROID DETECTION RESULTS

\section{Realization OF Pro Blocking SchedUling FUNCTION}

\section{A. Video speed measuring method}

The speed measuring device is implemented by means of formula (15).

$$
v=\frac{\Delta d}{\Delta t}=\frac{d_{1}-d_{0}}{t_{1}-t_{0}}
$$

Find the corresponding points, corresponding lines or corresponding blocks in the two frames. Ensure the video surveillance cameras is fixed. The spatial position and angle of the video surveillance cameras is fixed too. There is a corresponding comparability of the pixels in the two frames taken by the video surveillance cameras. As shown in Figure 8, located to the corresponding point or the corresponding block in the two image position $S_{1}$ (time frames $t_{1}$ ) and $S_{0}$ (time frames $t_{0}$ ), Subtract the two position can obtain the corresponding point or the pixels distance $\left(S_{1}-S_{0}\right)$ corresponding to moving in time $t_{1}-t_{0}(\Delta t)$. According to the formula above, the moving speed of the block in the image can be calculated naturally, the units is "pixels per second".

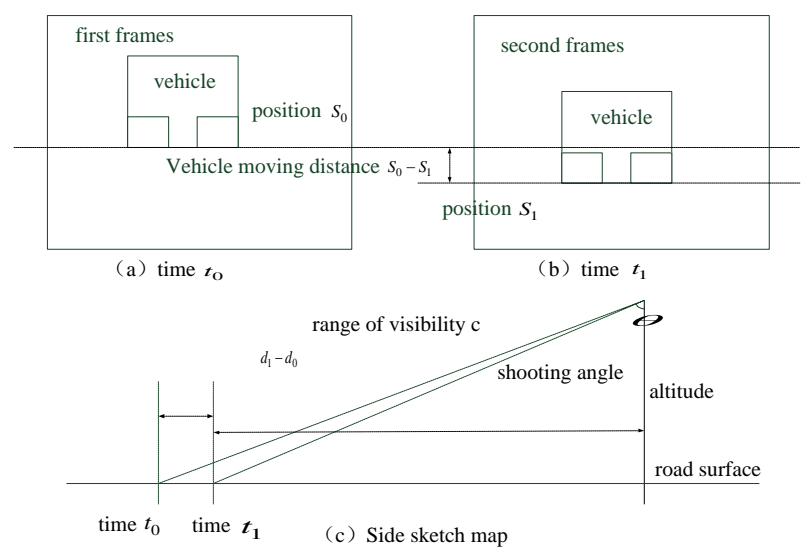

FIGURE VIII. TWO FRAMES OF IMAGES AT $t_{0}$ AND $t_{1}$ MOMENTS

As shown in figure 9, the image captured from the video signal, take the standard video signal resolution as an example, the height is 576 pixels and width is 768 pixels. For vehicle surveillance video, the width of the video field can choose just as the single lane width, usually about 3.5 meters.

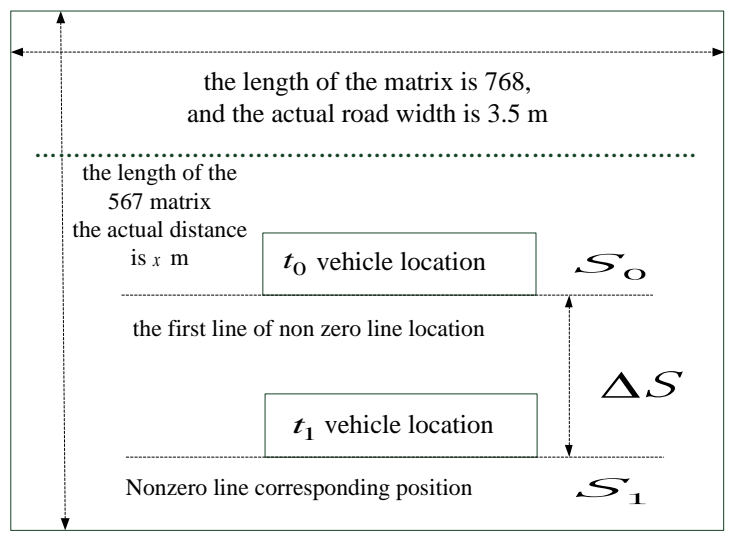

FIGURE IX. THE MOVEMENT OF A BLOCK IN A TWO FRAME IMAGE 
Within the scope of the road can be seen $x$ m meters in length, that is, the actual distance of the road represented by the video height is $x \mathrm{~m}$, then can get the formula (16).

$$
\frac{768}{3.5}=\frac{576}{x \cos \theta}
$$

By since the aforementioned visual effects are not taken into account, the vertical distance in the image can be considered equal, and the corresponding actual distance is equal, then can get

$$
\Delta S \cdot 576=\Delta d \cdot x
$$

Assuming the measured pixel distance is $\Delta \mathrm{S}$. The angle of video surveillance cameras is $\theta=60^{\circ}$ (the angle between the vertical direction and the camera angle used in the experiment).The $\Delta d$ and $\Delta t$ are known, so the speed can be calculated.

\section{B. Video vehicle counting design}

When the vehicle passes through the virtual coil, the corresponding area characteristics in the image will change. The vehicle can be determined to pass when the information in the virtual coil is detected to match the moving characteristics of the vehicle. Through the analysis of the characteristics of the change and the use of related algorithms for vehicle counting and speed extraction. It is necessary to set the appropriate location and size of the virtual coil in order to ensure the accuracy of vehicle detection. Usually, the virtual coil is set perpendicular to the direction of the road. And the coil is positioned near the end of the image. The width of the coil is approximately equal to the width of the single lane. The distance between two detection lines is about $1 / 3$ of car drivers. As shown in figure 10, each lane is set individually according to the angle in the video.

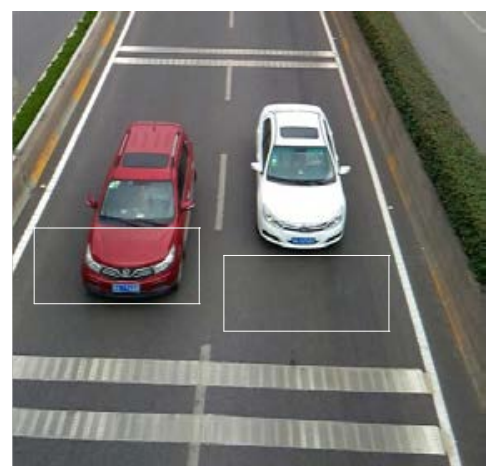

(a)
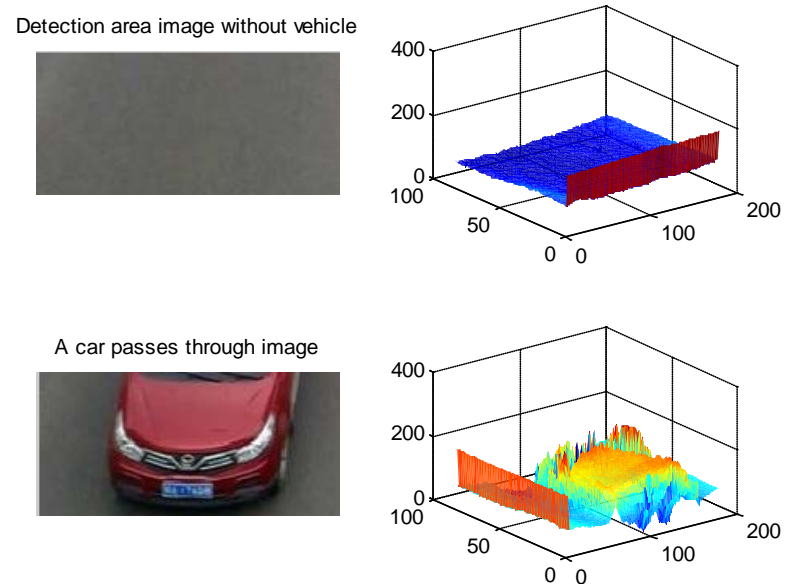

(b)

FIGURE X. THE SKETCH MAP OF DETECTION AREA AND ITS THREE-DIMENSIONAL DISPLAY OF GRAYSCALE

The gray value in the virtual coil is certain. The vehicle will pass through the detection area because of the sudden change of the vehicle passing, so that the vehicle can be counted. None vehicle passing and vehicle passing's gray scale 3D graphs are showing above.

\section{Scheduling Policy for Pre-blocking}

The situation where there is no taxi on the whole viaduct is very rare. But in order to prevent the situation from occurring, the first step is to determine whether the elevated road can extract relevant information of the speed of taxi. If not, count the vehicle through the main road in the unit time in order to determine the traffic flow of elevated road. A large number of data have proved that the elevated road from the sub-stable state of the upcoming congestion to the occurrence of congestion, the intermediate time is about 5-10 minutes, so in this time there can be pre-blocking scheduling. At the same time, after the first 5 minutes, when the traffic situation is judged to be congested, the dispatching center releases traffic information and dispatch information through the information system. The overall block diagram of the system is shown in figure 11 .

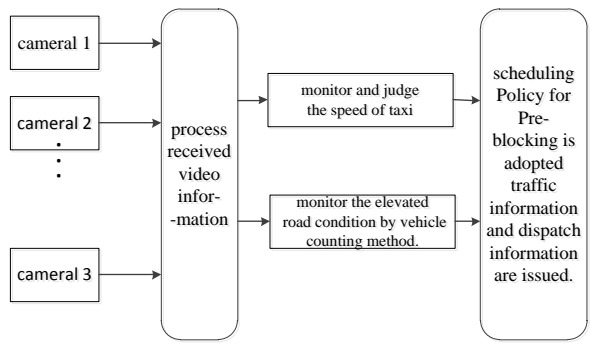

FIGURE XI. THE WHOLE DESIGN OF SYSTEM DIAGRAM

Take the vehicle count on the main road if the camera does not extract the information of the taxi. The critical density of the elevated road is $25 \mathrm{Veh} / \mathrm{Km}$ in single lane, and based on camera spacing thresholds are obtained. If the main road traffic flow exceeds the threshold, the time 30 seconds, count up and sort the uplink vehicles on all ramps. Turn off uplink ramps those are less than half of the total number of ramps in 
sequence. After 5 minutes, if the main traffic flows below the threshold, turn off the uplink ramp. Otherwise, close the specified uplink ramp. At the same time, the number of vehicles per unit time is counted until the congestion conditions are not satisfied. The software design flow diagram is shown in figure 12 .

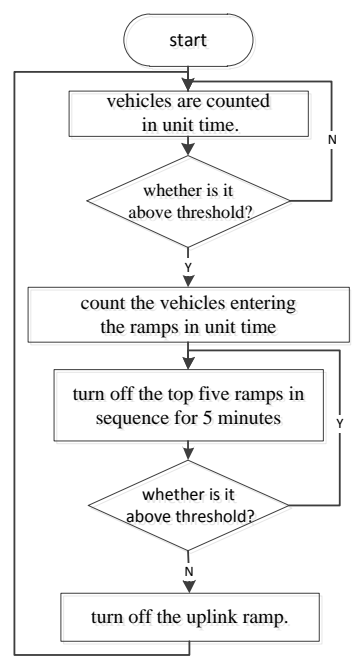

FIGURE XII. TAXI SOFTWARE DESIGN FLOW CHART WAS NOT DETECTED

If the camera extracts the speed of the taxi and the average speed $\left(V_{x}\right)$ of the taxi measured by each camera location are approximately equal, take $1 / 4$ as a threshold $V_{\min }$ of the design speed in the elevated road, when the $V_{x}$ bellows the $V_{\min }$, to determine the viaduct is about to occur congestion. At this point, count the vehicle on all ramps, for 30 seconds. Sort the difference $(\Delta)$ between uplink and downlink vehicles, and turn off the upper ramp less than half of the total number of ramps in sequence. If the average speed is above the threshold 5 minutes later, turn on the off uplink ramps. Otherwise, turn off the specified uplink ramps, and the average speed of vehicles is compared with the threshold in real time until the congestion condition is not satisfied. If the average speed of a taxi measured by a camera is depressed rapidly, the speed of the taxi extracted from the camera position behind the camera is used as the judging condition.

\section{CONCLUSION}

The viaduct system is an important part of city traffic. It is playing a positive role in alleviating traffic congestion. Based on a large number of investigations and researches, this paper selects the speed of viaduct taxies as the basis and the traffic counting as a supplement to judge the elevated road traffic congestion. This paper puts forward a feasible method for identification of the taxi, and the method is verified by simulation, in which the results prove the feasibility of this method. On this basis, this paper studies a response to elevated road congestion and disposal mechanism, and gives the flow chart of software design. The research on dispatching method of cutting congestion in elevated road is epochal which can be effectively applied to the traffic management in elevated road.

\section{REFERENCES}

[1] Nguyen H, Liu W, Chen F. Discovering Congestion Propagation Patterns in Spatio-Temporal Traffic Data[J]. IEEE Transactions on Big Data, 2016, PP(99):1-1.

[2] Liu W, Wu Q, Wen F, et al. Day-Ahead Congestion Management in Distribution Systems Through Household Demand Response and Distribution Congestion Prices[J]. IEEE Transactions on Smart Grid, 2014, 5(6):2739-2747.

[3] Sun Tao, Song Yanguo. Modeling and identification of flight dynamics system for rotorcraft [J]. Journal of Nanjing University of Aeronautics and Astronautics. 2011, (23): 387-392.

[4] Murphy C, Soroudi A, Keane A. Information Gap Decision TheoryBased Congestion and Voltage Management in the Presence of Uncertain Wind Power[J]. IEEE Transactions on Sustainable Energy, 2015, 7(2):841-849.

[5] Song Dalei, Qi Juntong, Han Jianda ect. Theoretical and experimental study on Modeling and active model control of rotorcraft flying robot system [J]. Acta Automatica Sinica, 2011, 37(4):480-495.

[6] Li Yawen, Zhai Jinling,Peng Xiaobang. Design and implementation of a position control PID four rotorcraft [J]. Automation and instrumentation, 2016(7):96-97.

[7] YANG Liben, ZHANG Weiguo, HUANG Degang. Robust trajectory tracking for quadrotor aircraft based on ADRC attitude decoupling control [J]. Journal of Beijing University of Aeronautics and Astronautics, 2015, 41(6): 1026 - 1033.

[8] Huang S, Wu Q, Cheng L, et al. Optimal Reconfiguration-Based Dynamic Tariff for Congestion Management and Line Loss Reduction in Distribution Networks[J]. IEEE Transactions on Smart Grid, 2016, 7(3):1295-1303.

[9] Shao C, Wang X, Shahidehpour M, et al. Partial Decomposition for Distributed Electric Vehicle Charging Control Considering Electric Power Grid Congestion[J]. IEEE Transactions on Smart Grid, 2016, 8(1):75-83.

[10] Oosedo,A,Abiko,S,Konno,A,Koizumi,T,Furui,T,Uchiyama,M,developm ent of a quad rotor tail-sitter VTOL UAV without control surfaces and experimental verification Robotics and Automation(ICRA),2013IEEE International Conference on,2013:317-322.

[11] A.Rodic, G.Mester, Modeling and Simulation of Quad-rotor Dynamics and Spatial Navigation, 2011 IEEE 9th International Symposium on Intelligent Systems and Informatics,2011.

[12] XU G H, MO Z. Modified adaptive flight control of quadrotor based on single neuron PID [C] //International Conference on Information Science and Technology (ICIST). Yangzhou, China: IEEE, 2013: 313-316.

[13] Koukoula D I, Hatziargyriou N D. Gossip Algorithms for Decentralized Congestion Management of Distribution Grids[J]. IEEE Transactions on Sustainable Energy, 2016, 7(3):1071-1080.

[14] Xu C, Zhao J, Muntean G M. Congestion Control Design for Multipath Transport Protocols: A Survey[J]. IEEE Communications Surveys \& Tutorials, 2016, 18(4):2948-2969.

[15] Kachroo P, Gupta S, Agarwal S, et al. Optimal Control for Congestion Pricing: Theory, Simulation, and Evaluation[J]. IEEE Transactions on Intelligent Transportation Systems, 2016, PP(99):1-7.

[16] Xiao M, Jiang G, Cao J, et al. Local Bifurcation analysis of a delayed fractional-order dynamic mdel of dual congestion control algorithms[J]. IEEE/CAA Journal of Automatica Sinica, 2016, PP(99):1-9.

[17] Jr A C N, Schwartz W R. A scalable and flexible framework for smart video surveillance[J]. Computer Vision \& Image Understanding, 2016, 144(C):258-275.

[18] Bhushan Nemade. Automatic Traffic Surveillance Using Video Tracking[J]. Procedia Computer Science,2016,79: 